\title{
Trapping Dynamics of Diindenoperylene (DIP) in Self-Assembled Monolayers using Molecular Simulation
}

\author{
Ananth P Kaushik and Paulette Clancy \\ School of Chemical and Biomolecular Engineering, Cornell University \\ Ithaca, NY 14853
}

(Dated: November 7, 2010)

\begin{abstract}
All-atom Molecular Dynamics simulation methods employing a well-tested intermolecular potential model, MM3, demonstrate the extent of the propensity for diindenoperylene (DIP) molecules to insert between molecules of a self-assembled monolayer (SAM) during a deposition process intended to grow a thin film of this organic semiconductor molecule onto the surface of self-assembled monolayers. The tendency to insert between SAM molecules is fairly prevalent at normal growth temperatures and conditions, but is most strongly dependent on the density and the nature of the SAM. We posit the existence of an optimal density to favor surface adsorption over insertion for this system. DIP is less likely to insert in fluorinated SAMs, like FOTS, than its unfluorinated analog, OTS. It is also less likely to insert between shorter SAMs (e.g., less insertion in OTS than ODTS). Very short length, surface-coating molecules, like HDMS, are more likely to scatter energetic incoming DIP molecules with little insertion on first impact (depending on the incident energy of the DIP molecule). Grazing angles of incidence of the depositing molecules generally favor surfaceadsorption, at least in the limit of low coverage, but are shown to be dependent on the nature of the SAM. The validity of these predictions is confirmed by comparison of the predicted sticking coefficients of DIP at a variety of incident energies on OTS, ODTS, and FOTS SAMs with results obtained experimentally by Engstrom et al. The simulation predictions of the tendency of DIP to insert can be explained, in large part, in terms of binding energies between SAM and DIP molecules. However, we note that entropic and stochastic events play a role in the deposition outcomes.
\end{abstract}

PACS numbers:

\section{INTRODUCTION}

Self-assembled monolayers (SAMs) consist of a layer of functionalized long-chain molecules tethered to a solid substrate. Their presence as a "coating" on a surface is attractive in a number of applications due to the possibility they provide to tune the properties of the surface by selectively modifying functional groups on the SAM $[1,2]$. SAMs of organosilane molecules are of particular technological interest in organic electronics because they can be assembled on hydroxylated surfaces such as $\mathrm{SiO}_{2}$ for applications in areas such as organic electronics, electronic sensors and biosensors [3, 4]. Not surprizingly then, they have been the subject of extensive theoretical and experimental research due, for instance, to their ability to improve the mobility of organic thin films for electronic devices, presumably by improving surface order.

There have been several molecular-level computational studies of SAMs, some of which are particularly relevant to the studies in this paper [1,4-10]. Yamamoto et al. studied the influence of hydrogen bond conformations of alkylsilane SAMs using molecular mechanics and Molecular Dynamics simulations [1]. A study of the diffusion of tricresyl phosphate (TCP) molecules on an octadecyltrichlorosilane SAM [4] found that the TCP molecules are highly mobile on the surface with a small isotropic diffusion activation barrier of about $0.1 \mathrm{eV}(9 \mathrm{~kJ} / \mathrm{mol})$. The TCP molecules prefer to diffuse over the surface rather than become inserted between the SAM molecules. The authors detected the presence of an anisotropic energy barrier in the vicinity of a vacancy defect. Close to the defect, TCP molecules became trapped as part of the molecule became embedded within the vacancy created by the SAM molecules. TCP molecules were observed to approach the defect against (rather than along) the tilt of the SAM molecules.

The structural properties of alkanethiol SAMs have been determined as a function of temperature, lattice spacing (density), and molecular chain length [5]. For instance, chains containing 13 carbons tilt from the surface normal by a collective angle of $25^{\circ}$ along the next-nearestneighbor direction at $300 \mathrm{~K}$. The tilt angle can vary by as much as $20^{\circ}$ for a temperature increase of $200 \mathrm{~K}$, and change by $30^{\circ}$ for a lattice constant increase of $0.6 \AA$. There have also been studies of hyperthermal deposition of inert gas atoms on SAM surfaces: Simulations of Ar, Xe and Ne on SAMs showed inelastic scattering and trapping dynamics $[4,11-14]$. Xe, in particular, showed a sort of directed ejection mechanism after insertion into the SAM matrix [15].

Experimentally, a variety of preparation methods are available for self-assembled monolayers and multilayers of alkyltrichlorosilanes, including studies experimenting with different specialized functional groups in order to get specific desirable properties [16]. There have also been studies aimed at controlling surface properties by varying the alkyl chain lengths of the SAMs to enhance the electrical performance of field-effect transistors (FETs) of different organic molecules [17]. Effects of alkyl chain lengths of SAMs on the film growth of organic molecules like pentacene have been studied. In particular, Bao et 
al. have observed that the nature of film growth, and hence the performance of transistors, is significantly affected by the alkyl chain of the SAM molecule being odd or even in length and by the density of the SAM [18-20].

Despite these prior computational studies of the characteristics of SAMs in contact with a diffusing surface atom or molecule, there has been no previous study of the deposition of organic thin films on SAM surfaces, either at thermal or hyperthermal deposition conditions. Importantly for the work to be presented here, the SAM surfaces that have been studied previously have always been well packed; there have been no studies involving low packing densities of SAMs. However, experimental studies, at least on amorphous $\mathrm{SiO}_{2}$ surfaces, are often performed with a low density of SAM molecules and thus the ability to simulate deposition of molecular species on low-density SAM surfaces is of great importance in the study of the trapping dynamics of organic molecules on SAMs. In this study, we considered three molecules that are capable of creating self-assembled monolayers (fluorooctatrichlorosilane (FOTS), octatrichlorosilane (OTS) and octadecyltrichlorosilane (ODTS)). In addition, we looked at the behavior of hexamethyldisilazane (HMDS), a short-chain, surface-coating, ligand which provides an interesting contrast to the SAMs. These four molecules (three SAMs and HMDS) are used as the surface upon which an organic semiconducting molecule, diindenopyridine (DIP), is to be grown as a thin film; see Fig. 1 . The SAM molecules have long-chain carbon backbones terminated at one end by a methyl group (a fluorinated methyl group in the case of FOTS) and at the other end by $\mathrm{SiCl}_{3}$. HMDS has a very short (two-carbon) chain. The FOTS and the OTS molecules have an 8-carbon backbone and the ODTS has an 18-carbon backbone; again, see Fig. 1.

By "trapping dynamics" we are referring to mechanistic processes which lead to the inadvertent trapping of deposited molecules between SAM molecules on the surface (Fig. 2(a-c)), as opposed to surface-adsorption events which are the usual intent of a deposition process (Fig. 2(d-f)). As shown in Fig. 2(g-i), depositing molecules can also be "scattered," that is, they hit the surface, or are inserted into the SAM, but ultimately are expelled from the vicinity of the SAM without inclusion either on the surface or between SAM molecules. Whether "trapping" of molecules between SAM molecules is disadvantageous or not is not clear: At first glance, the unintended trapping process seems likely to lead to problems with the creation of an ordered surface (depending, perhaps, on the length of the deposited molecules in relation to that of the SAM molecule). But it could also be speculated that the insertion process could lead to an effectively higher density SAM monolayer, perhaps one that enhances surface deposition, and hence be essentially a benign process in terms of the quality of film growth that can occur subsequently. We will investigate some of these issues here.

In this study, we have performed Molecular Dynamics
(MD) simulations to characterize the trapping dynamics of diindenopyridine (DIP) on different commonly studied SAM surfaces and to identify the major factors that affect the growth of DIP thin films on SAMs. The motivation for performing a molecular simulation study is that it allows an elucidation of the mechanisms of trapping in greater detail than is possible from experiments alone. In addition to studies that replicate the conditions of energetic deposition experiments by the Engstrom-led group at Cornell, molecular simulations also afford the chance to investigate trapping dynamics in regimes that are difficult or impossible to study experimentally: the effect of packing density, orientation of the incident DIP molecule and, to some extent, temperature on deposition outcomes fall into this category. In this paper, we will identify several key parameters which affect the trapping of DIP and which could be tuned favorably in order to increase the efficiency with which SAMs are able to affect the ordered growth of DIP molecules (and, by extension, other small organic semiconductor molecules).

One of the advantages of a computational study of this kind is the ability to observe deposition events at a molecular scale. This gives us an opportunity to calculate, accurately, the outcome of a single deposition event without having to perform a post-mortem analysis once the bulk film has been deposited. In experiments, it is possible to calculate the fraction of DIP molecules which are not scattered from the SAM surface, but it is essentially impossible for experiments to differentiate between the fraction that becomes inserted into the surface rather than becoming adsorbed on the surface. Such knowledge is potentially important since the fate of depositing molecules governs both the nature and quality of the bulk film grown. This study is intended to capture details of deposition events, some of which may not be accessible experimentally, in order to explain a few key aspects that affect the observed bulk properties of the deposited film.

\section{A. Configuration of the system}

In the experimental set up used by Engstrom et al. $[21,22]$, molecules that form surface coatings, like the SAMs and HMDS, are deposited on an amorphous silicon dioxide substrate. The head group, consisting of three $\mathrm{Cl}$ atoms, reacts with the $-\mathrm{OH}$ groups of the substrate, forming an -O-Si bond that tethers the molecule to the surface. In the simulation, we simply tethered each SAM molecule to an $\mathrm{x}-\mathrm{y}$ location on an undefined substrate surface. Since the ligands are relatively long, an explicitly modeled surface beneath the SAM proved to be unnecessary. The FOTS, OTS and ODTS SAM molecules were tethered to hexagonal lattice points in free space at the oxygen atom, i.e., the position of the oxygen atom was fixed throughout the simulation. This choice was made to follow experimental evidence which suggests that the oxygen atoms are attached to the substrate surface, thereby anchoring the SAM molecules at 
this point. It is possible that, on real surfaces, the SAM molecule could be tethered via more than one oxygen atom in the head group, which implies that the silicon atom of the head group is also essentially fixed in place [1]. To study the impact of this eventuality and better define an appropriate initial system configuration, we conducted simulations in which both the silicon and oxygen atoms were fixed in place. We found that the results, as measured by the energetics of the system, differed by less than $5 \%$ in energy and with no observable structural difference in the system whether just oxygen, or oxygen and silicon, were fixed. Thus all the remaining simulations described in this paper were performed assuming that the SAM molecule is tethered to the substrate by one oxygen atom.

For the short HMDS ligand we could not use the same approach since, at the lower densities considered here, incoming DIP molecules could readily move through gaps in the $\mathrm{x}-\mathrm{y}$ plane where the substrate should be located. To avoid this, we modeled the surface beneath the HDMS as the (111) face of a Si crystal. HMDS molecules were arranged on a square lattice appropriate for the underlying Si lattice and the density of the HMDS was chosen to be 3.24 molecules $/ \mathrm{nm}^{2}$. The effect of changing the density of all three tested SAMs on the trapping dynamics is explored below.

The simulation set up involved creating a hexagonal lattice of 98 SAM molecules, consisting of $7 \times 7$ unit cells with two molecules per unit cell. For FOTS and OTS, this involves the consideration of 2,940 atoms (and 5,928 atoms for the longer ODTS molecule). The choice of a hexagonal lattice was arbitrary, but it is convenient as a close-packed lattice and is one that has been the usual choice in prior simulations of SAM monolayers $[4,18]$. The characteristic lattice parameter of the hexagonal packing was chosen to match the packing density determined from X-ray reflectivity measurements [23]. The densities of the SAM surfaces were estimated from the experiments to be 2.0 molecules $/ \mathrm{nm}^{2}$ for FOTS, 2.75 molecules $/ \mathrm{nm}^{2}$ for OTS, and 2.83 molecules $/ \mathrm{nm}^{2}$ for ODTS.

The molecules were created using the Molden software package [24] and an energy minimization of the initial guessed structures was performed using a standard minimization algorithm - the limited memory L-BFGS minimization using a modified version of the algorithm of Nocedal which is a part of the TINKER software package [25]. The lengths of the SAMs, measured from the center of the oxygen atom to the center of the top carbon atom, were found to be $11.12 \AA$ for FOTS and OTS; the length of the ODTS molecule was $22.96 \AA$. The optimized structures of the five molecules are shown in Fig. 1.

\section{B. Intermolecular Potential Models}

The choice of intermolecular potential model is a very important and defining part of molecular simulation. We chose to use the non-reactive semi-empirical MM3 potential to model all the SAM-SAM and DIP-SAM interactions. There are no DIP-DIP interactions to consider here, as we studied the fate of a single depositing DIP molecule. The MM3 potential has been shown, by us and others [6-9], to accurately describe hydrocarbons [6], fluorinated hydrocarbons [8] and multiplyringed molecules of the type we studied here. MM3 incorporates stretching, bending, and torsional energies, as well as the van der Waals interaction energies based on phenomenologically determined parameters. We have used this model extensively to study the energetics and structural characteristics of an array of small organic molecules including the acenes, rubrene, DIP, sexiphenyl and $\mathrm{C}_{60}[9,10]$ and have confidence in its ability to model conjugated systems. Our most recent study involved an extensive survey of twelve Density Functional Theory models, as well as the MM3 and MM3- $\pi$ models for biphenyl and eight models (four DFT and four semi-empirical models) for the sexiphenyl molecule. We found virtually all the models to give consistent energetically preferred structures [26]. Both MM3 models (with and without an additional term to represent pi-bonding) represented the behavior of sexiphenyl and biphenyl molecules with quantitative accuracy compared to the DFT models. Based on our studies and those of the Allinger group, we are confident in the ability of MM3 to be sufficiently accurate to capture the fate of a single DIP molecule on a SAM surface without having to resort to using MM3- $\pi$, a variant of MM3 which is more accurate for molecules with an extensive $p i$-electron system but which is about 20 to 100 times slower in execution time.

\section{Simulation Details}

The time evolution of the system was followed using a Molecular Dynamics simulation approach using the Modified Beeman Algorithm as part of the TINKER software package. As mentioned above, optimized structures of the SAM molecules and the DIP molecule were obtained from an energy minimization of an initial guess structures using a standard minimization algorithm, here, the limited memory L-BFGS minimization using a modified version of the algorithm of Nocedal [27]. The system was first thermalized at $300 \mathrm{~K}$ using a Nosé-Hoover thermostat in the canonical (NVT) ensemble for a period of $50 \mathrm{ps}$ with a time step of $1.0 \mathrm{fs}$ (i.e., 50,000 time steps) in order to suppress any significant fluctuations in temperature and equilibrate the system of SAM molecules (before the deposition of the DIP molecule). Anticipating a result described more fully below, the simulated value of the film thickness was found to be within one standard deviation of the experimentally calculated value which helps to justify the choice of hexagonal packing of the SAM molecules, though it does not preclude another geometry from working equally well. 


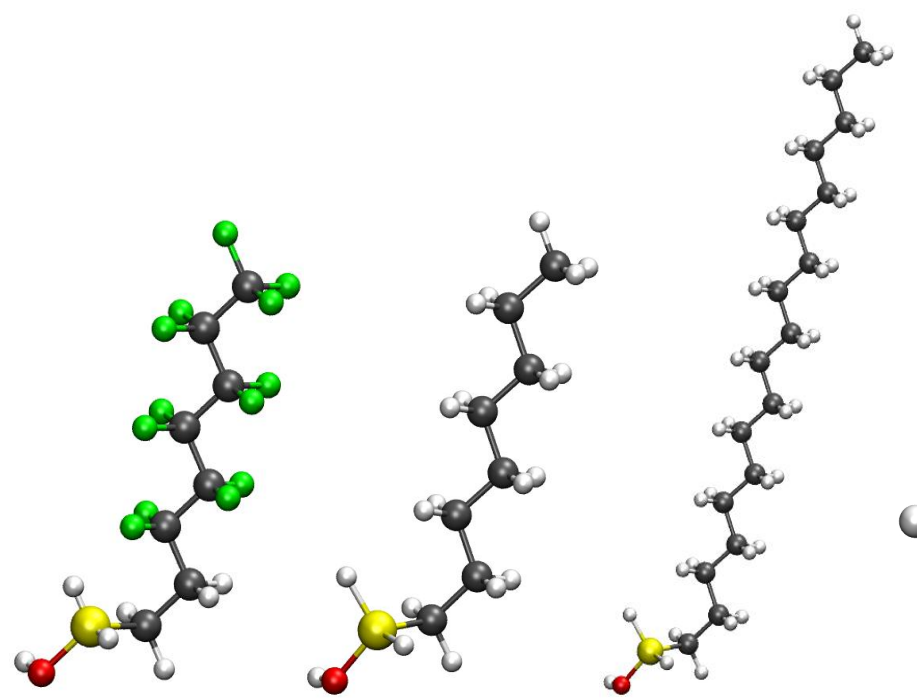

(a) FOTS (b) OTS

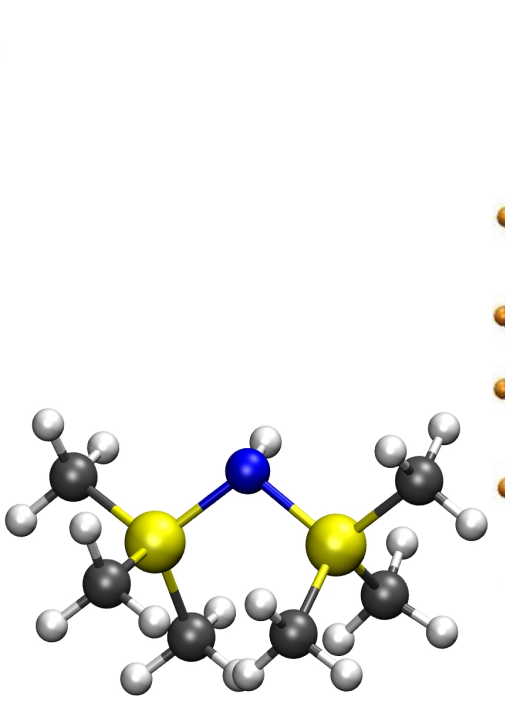

(d) HMDS

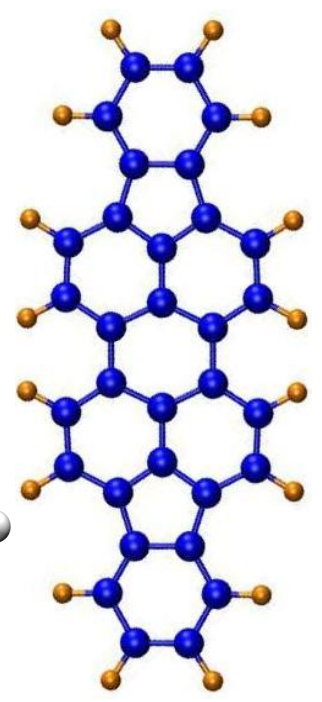

(e) DIP

FIG. 1: Molecular models of the molecules.

Since the consideration of each additional DIP molecule adds another 48 atoms to the system, following the deposition process of DIP molecules onto the SAM surfaces quickly becomes computationally very expensive. That being the case, the simulations in this paper were restricted to the deposition of only one molecule of DIP on the SAM surface. The DIP molecule was given a random orientation at a height of $30 \AA$ above the SAM surface in 3D space and given a random initial ( $\mathrm{x}-\mathrm{y})$ coordinate above the surface of the SAM. Simulations were performed at six different incident energies of the DIP molecule to match the incident energies used in Engstrom's experiments [23]. Simulations of collisions between the DIP molecule and the SAM surface were carried out in the microcanonical NVE ensemble to avoid any unwanted bias due to velocity corrections used to scale the temperature to a desired value in the canonical ensemble. The effect of using NVE versus NVT has been tested for a related system, pentacene, and no issue has been found [9].

Each simulation in our study constitutes an individual deposition event. Simulations were performed for a period of $25 \mathrm{ps}$ with a time step of $0.5 \mathrm{fs}$ (50,000 time steps). This time frame was chosen because it was found to be sufficient for the molecular collision to occur between DIP and the SAM surface and for the system to then thermalize to its original state. Each simulation (at a given incident energy of the DIP molecule and SAM density) was carried out 100 times, each time with a different initial random orientation of the DIP molecule, so that we could gather enough statistics to accurately determine the probability of the DIP molecule sticking to the SAM surface. An alternative view of this set of 100 simulation runs is that we studied the low-coverage limit of DIP sub-monolayer growth for 2.5 ns at a deposition rate of $4 \times 10^{10}$ molecules/second (in which no DIP was close enough to encounter another DIP molecule). We report the "sticking fraction" as the ratio of the number of events that led to a particular outcome (adsorption on the surface, insertion, scattering) divided by the number of events studied (here, 100).

The outcome of each DIP collision with the SAM surface was recorded and observed to fall into one of the following categories: The DIP molecule can deposit on top of the SAM monolayer (the experimentally intended outcome), insert itself between SAM molecules, or collide with the SAM and bounce off (a scattering event). The same process was carried out for monolayers of the SAMs and HMDS at six different incident energies matching the experimental values $[23](E=1.5,5.07,7.69,9.0,10.0$ and $12.31 \mathrm{eV}$ ). Three additional studies were carried out for the SAMs to look at the effect of varying the packing density (six densities from 2.0 to 4.0 molecules $/ \mathrm{nm}^{2}$ ), the temperature (five temperatures from $200 \mathrm{~K}$ to $400 \mathrm{~K}$ ) and with different initial orientations of the DIP molecule (with the long molecular axis perpendicular to the surface, parallel to the surface and at orientations in between) to investigate the nature of the sticking of DIP on the SAM surfaces. Thus each SAM molecule was studied in about two thousand different simulations.

\section{Results}

After thermalization to $300 \mathrm{~K}$, the initially vertically oriented SAM molecules were observed to lean over to attain a minimum energy configuration, adopting a preferred angle with the surface normal. This translates to an observed film thickness that is smaller than the total length of the molecule, measuring film thickness as the 


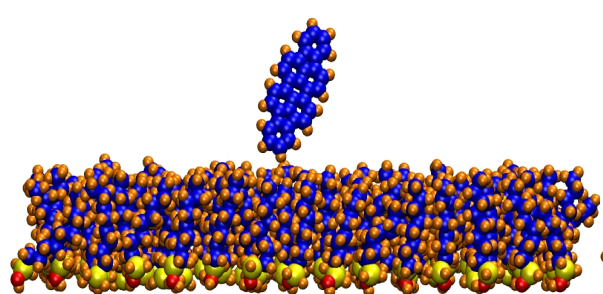

(a) Approach

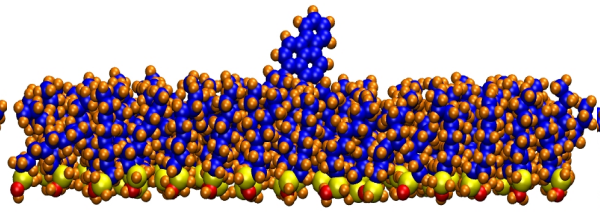

(b) Collision

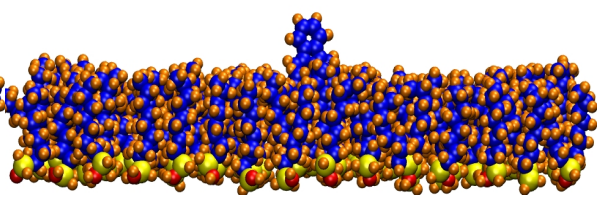

(c) Insertion

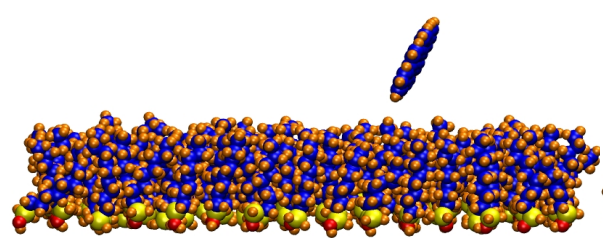

(d) Approach

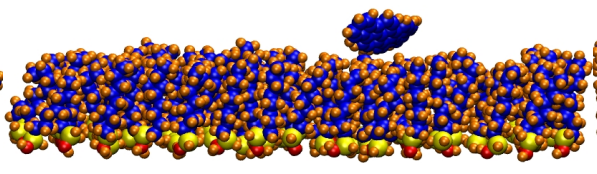

(e) Collision

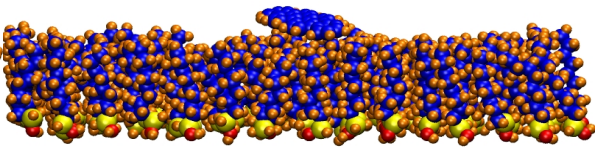

(f) Adsorption

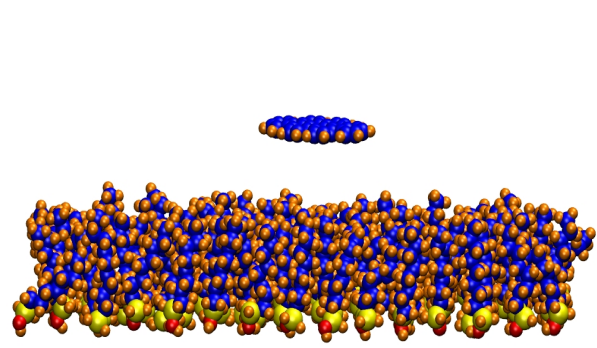

(g) Approach

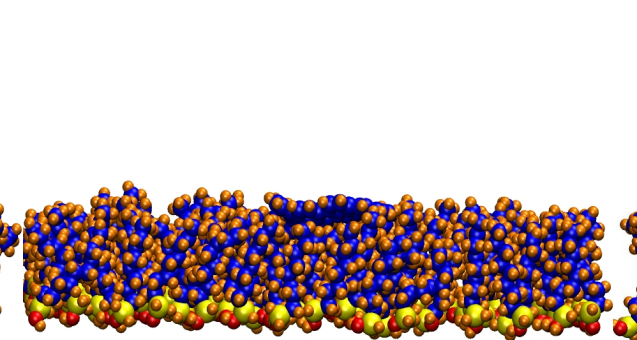

(h) Collision
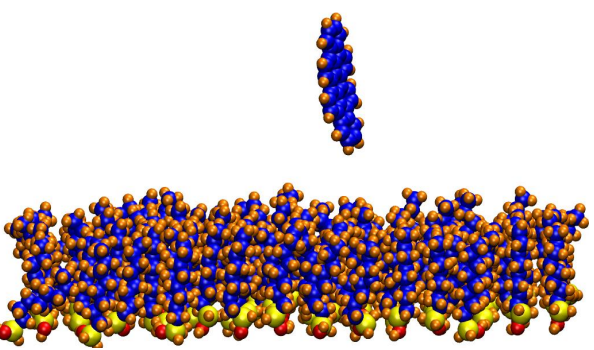

(i) Scattering

FIG. 2: Possible events of DIP collision: (a-c)Insertion event, (d-f)Adsorption event and (g-i)Scattering event.

vertical distance from the silicon atom to the top carbon atom. Table I compares the average film thicknesses of the different SAM surfaces obtained from experiments (XRD data) [23] and simulation, showing agreement that is typically within a standard deviation of the experimental data. What is less easy to capture in a quantitative way is the dynamics of the system realized in simulation movies of the system. We observed the SAM molecules to move in a random waving motion, akin to tall grass blowing in the wind. The FOTS SAM adopts a much more ordered configuration compared to the OTS SAM. This may be explained by stronger interactions between the molecules in the FOTS SAM in comparison to the OTS SAM, making the FOTS SAM appear "stiffer" than OTS. Not surprizingly, the longer ODTS SAM was found to be much more flexible than the other two SAMs.

Data from these simulations show that the probability that a DIP molecule will become adsorbed on the surface of the SAM depends on several factors, each of which can affect deposition and, in turn, affect the quality of the film of DIP on the SAMs. Some of these factors are considered in greater detail in the following sections.

\section{E. Sticking coefficient of DIP on SAMs}

We began by calculating the sticking coefficient of DIP as the fraction of molecules that are not scattered from the surface of the SAM. This value includes the fraction of DIP molecules that get adsorbed on the surface of the SAM and the fraction that insert into the SAM surface; this is essentially the quantity measured in experiments. The sticking fraction was calculated as the fraction of

TABLE I: Film thickness of the SAM observed in experiments and by simulation. The computed tilt angle is expressed as being measured from the surface normal.

\begin{tabular}{cccc}
\hline \hline Surface & Experiment $(\AA)^{a}$ & ${\text { Simulation }(\AA)^{a}}^{a}$ & Tilt Angle $\left(^{\circ}\right)$ \\
\hline FOTS & $6.32(0.5)$ & $7.5(1.1)$ & 48 \\
OTS & $6.27(0.65)$ & $7.9(1.4)$ & 45 \\
ODTS & $17.31(1.75)$ & $17.5(2.7)$ & 40 \\
\hline \hline
\end{tabular}

${ }^{a}$ The values in parentheses indicate the standard deviation from the average values. 
non-scattered molecules in 100 trial depositions, as described in the previous section. The final coordinates of the molecules were recorded to determine the outcome of the deposition event (scattered, adsorbed, or inserted). The variation of the sticking coefficient with different incident energies of the DIP molecule on the three SAMs and HMDS is shown in Fig. 3.

As seen in Fig. 3, the experimental and simulated results follow a similar trend and are in fairly good quantitative agreement given that sticking coefficients can vary by many orders of magnitude on different surfaces. Thus there was no guarantee, a priori, that the simulation predictions would be within an order of magnitude of the experimental results. Both experiment and simulation predict that the overall sticking coefficient decreases with increasing energy of incidence of the DIP molecule.

In order to remain on the surface of the SAM, the incoming DIP molecule has to lose some or all of its kinetic energy upon collision. The energy of the incident molecule will be dissipated into the lattice (through lattice vibrations in the form of surface phonons) and the torsional motion of the SAM molecules. Since the area of the SAM surface is large compared to the DIP molecule, we have observed that the temperature of the SAM surface is largely unaffected by the appearance of the DIP molecule (a maximum temperature variation of $\pm 5 \mathrm{~K}$ occurs at the moment of impact and the surface returns quickly, within picoseconds, to the equilibrium temperature). Thus, with increasing incident energy of the DIP molecule, the harder it becomes for the SAM surface to absorb and dissipate the energy. This means that there is an increasing tendency for the DIP molecule to retain a significant part of its incident energy after collision with the SAM and, consequently, for the DIP molecules to be ejected from the SAM as a scattered molecule.

The same decreasing trend to stick on the surface with increasing incident energy is seen on all SAM surfaces: The tendency to stick is highest for ODTS, next for OTS, and the least for FOTS. The tendency to remain on the surface after the first collision is almost zero for HMDS at energies above about $6 \mathrm{eV}$; we shall return to an explanation of the HDMS results later in this section. We shall show below that factors such as the length of the SAM molecule, the interaction energy of SAM with DIP, and the packing density of the SAM molecules, all contribute to these observed trends of the sticking fraction of DIP with increasing incident energy.

Our observations of hundreds of simulations of the way that DIP molecules interact with SAM surfaces has led to some overall conclusions about the tendency of DIP molecules to adsorb on the surface of the SAM rather than insert themselves into the SAM surface or scatter. Depending on the position of incidence and the local structure of the SAM surface, the DIP molecule has the opportunity to: (1) collide with one SAM molecule, (2) collide with more than one SAM molecule, or (3) land in the interstitial space between the SAM molecules. The more SAM molecules with which the DIP molecules

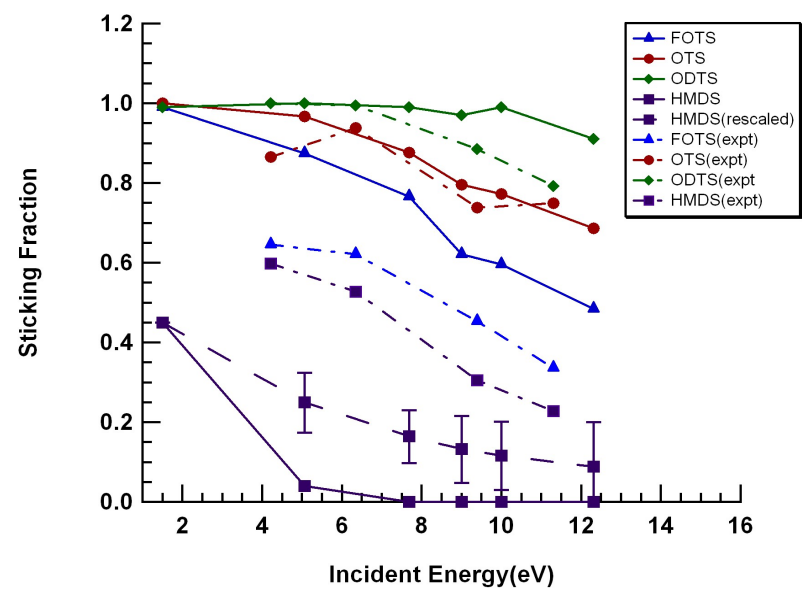

FIG. 3: Overall sticking coefficients of DIP on four different SAMs as a function of incident energy at room temperature. Packing density: $\mathrm{FOTS}=2.5, \mathrm{OTS}=2.75$, $\mathrm{ODTS}=2.83$ and $\mathrm{HMDS}=3.4$ molecules $/ \mathrm{nm}^{2}$. Symbols joined by lines are simulation results. Dot-dashed lines correspond to experimental values [23]. The dashed

line labelled as HMDS(rescaled) shows the sticking coefficient if the effect of a second collision of DIP with the HMDS surface is added.

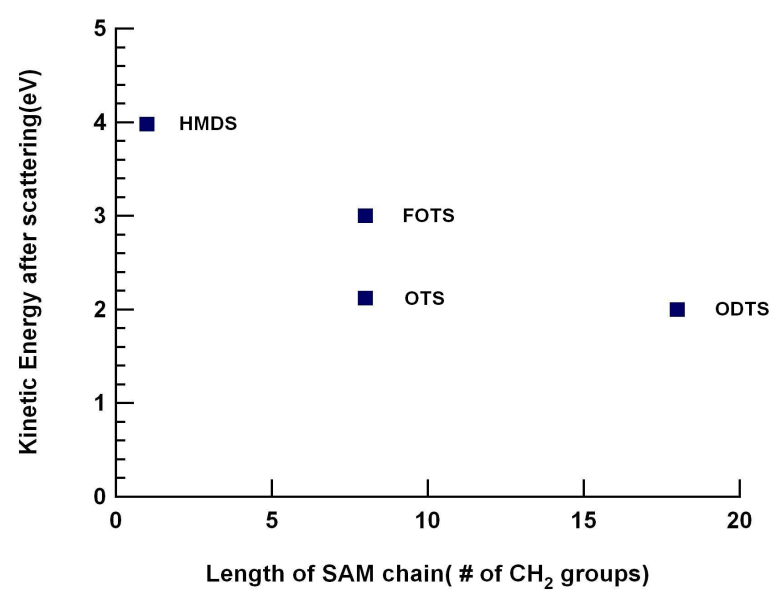

FIG. 4: Exit kinetic energy of DIP on different surfaces for an incident energy of $7.7 \mathrm{eV}$.

comes in contact, the easier it becomes to dissipate its incident energy into the lattice. As mentioned above, the collective motion of the three SAMs we studied exhibited a wave-like motion, behaving like elastic springs tethered to an underlying substrate. Thus, if a DIP molecule collides with a single SAM molecule, this elasticity is capable of tossing it away from the surface. In practice, however, this effect is mediated by factors such as the orientation of the DIP molecule as it collides with the SAM molecule (e.g., impact parameter), the proximity of the other SAM molecules around it (to affect the local density), the energy of interaction between the SAM 


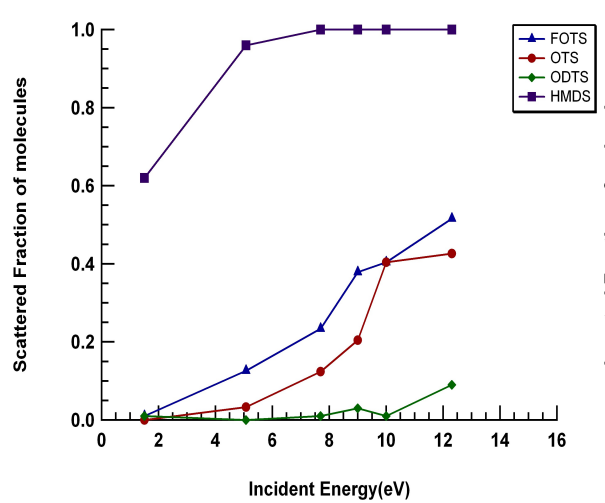

(a) Scattered fraction

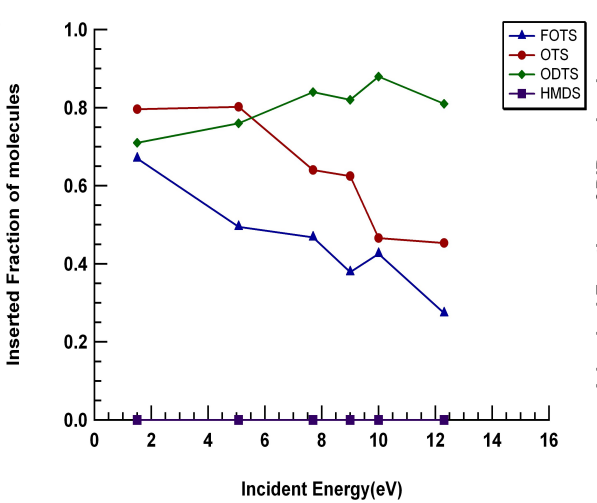

(b) Inserted fraction

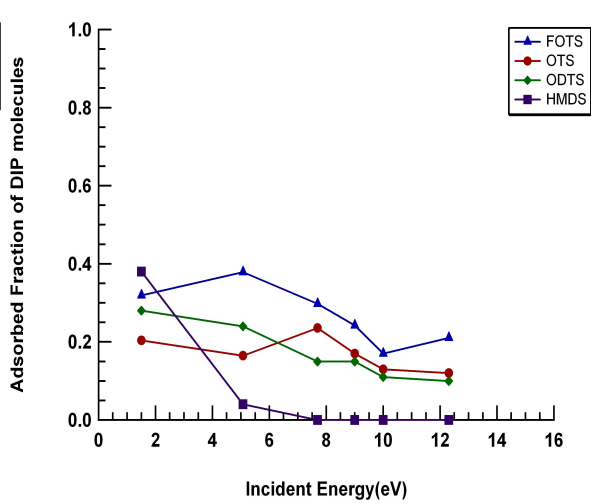

(c) Adsorbed fraction

FIG. 5: Deposition of DIP on FOTS, OTS and ODTS at different incident energies showing (a) scattered (b) inserted and (c) adsorbed fractions. Packing density: FOTS $=2.5$, OTS $=2.75$ and ODTS $=2.8$ molecules $/ \mathrm{nm}^{2}$.

molecule and the DIP molecule, and -of course- the incident energy itself. At low incident energies, a single SAM molecule may be able to absorb and dissipate the energy. But, at higher incident energies, it is likely that it will eject the DIP molecule. Collision with more than one SAM molecule is certainly more effective for energy dissipation, but the outcome of a given collision still depends on the factors described above. Molecules which are able to successfully dissipate enough incident energy so that the remaining energy is less than the binding energy between the DIP molecule and the SAM surface are adsorbed on the surface.

If a DIP molecule lands in the space between the SAM molecules, it invariably leads to a direct insertion event in which the energy of interaction between the DIP molecule and neighboring SAM molecules is strong enough to hold the DIP molecule embedded in the lattice. Steric hindrance could also be a contributing factor, preventing the ejection of the DIP molecule after insertion. Even comparatively long simulation runs, of the order of a nanosecond (2 million time steps), showed no tendency of the DIP molecule to desorb from its interstitial position once inserted into the lattice. More insight into this tendency for DIP to remain inserted within the SAM will be evident when we consider binding energies in a later section.

The other possibility as a route to insertion events (found more commonly at lower incident energies) is that the DIP molecule could get initially adsorbed on the surface, diffuse across it and then undergo insertion. The waving motion of the SAM molecules can give rise to situations where the diffusing DIP molecule encounters an energy "well" caused by the instantaneous parting of two SAMs. In this situation, it is energetically favorable for the DIP molecule to insert since the interaction energy between the SAM molecules and the DIP molecule is higher than that for surface-adsorption, simply because there are more interaction sites.

Other factors were observed to govern the sticking co- efficient, including the length of the SAM molecules and differences in interaction energies between DIP and different SAMs. Length affects the sticking probability: The data in Fig. 3 clearly show that the sticking fraction is highest for the longest molecule, ODTS, and least for the shortest one, HMDS, for all incident energies. However, the governing mechanism seems to be different in each case. In the case of ODTS, the length of the SAM is the strongest factor affecting the sticking fraction. Since the backbone of the ODTS molecule is very long, it exhibits a greater degree of flexible motion compared to the other SAMs, thus creating a greater amount of interstitial space for insertion to take place. Most of the incident DIP molecules undergo insertion into the very flexible matrix of ODTS, leading to a low adsorbed fraction, as shown in Fig. $5 \mathrm{c}$ and an even lower scattered fraction (see Fig. 5a). Indeed, the fraction of DIP molecules that insert into ODTS is observed to go up with increasing energy, which accounts for the very high overall sticking coefficient. Thus, having a higher overall sticking coefficient may not be the best criterion to judge surface adsorption since this value will include insertion events. OTS and FOTS molecules show little variation in the fraction of molecules that adsorb on the surface, but the tendency to insert DIP in these two SAMs decreases steadily with increasing energy (see Fig. 5b), and an increasing tendency to scatter (see Fig. 5a), leading to an overall decrease in sticking with energy observed in Fig. 3. As we shall show in the next section, differences in sticking behavior between the identically long FOTS and OTS molecules arise due to the chemical nature of the molecules and the packing density of the SAMs.

The reason for the precipitous fall in sticking coefficient of DIP on HDMS with increasing energy was harder to understand. Engstrom's experimental data show a decline in sticking on HMDS relative to the SAMS, like the simulation results, but they do at least show some sticking at higher energies that the simulation is unable to match (simulation predicts no sticking at all above $6 \mathrm{eV}$ 
after the first collision with the surface!). Having eliminated possible procedural effects and with no reason to single out the potential energy function for the HMDS model as being at fault, the most likely reason is the inability of energetic DIP molecules to "scrub off" enough energy to either adsorb or insert as a result of the first collision on the short stubby HMDS surface, which is what we are measuring in the simulation. We are ignoring the fact that DIP molecules could, and probably do, collide multiple times with the relatively "hard" HDMS surface before adsorbing or inserting. It seems reasonable that this effect will become more pronounced for higher incident energies. Considering only the first collision with the HMDS surface precludes access to a molecular mechanism open to the longer SAMS wherein the incoming energetic DIP molecules lose energy to the accommodating, more elastic, SAM molecules.

For the "unforgiving" HMDS surface, we suggest that it is important to follow the fate of individual molecules as they make multiple collisions with the surface. But the scope of such a study, following every scattered molecule as it traverses the surface, is inaccessible from a computational resource point of view. To compensate for this inability, we estimated the sticking coefficient after a second collision with the surface. For a given incident energy, we measured the energy of the DIP molecule as it left the surface for all 100 attempts and then averaged them to find a mean exiting energy. For example, a scattered DIP molecule initially having a $5 \mathrm{eV}$ incident energy is, on average, likely to leave the surface with an energy of $3 \pm 0.6 \mathrm{eV}$. DIP molecules with $12 \mathrm{eV}$ incident energy which scattered from the surface left with a mean energy of around $4 \pm 1.0 \mathrm{eV}$. If we assume that the DIP molecule has the opportunity to collide once more with the HMDS surface, we know from our measurement of its "exit" energy (which becomes its new incident energy for a second collision with the surface) its probability of sticking on the surface by interpolating data from the sticking fraction corresponding to that incident energy based on our data for first surface collisions. We then simply add this additional, second collision, contribution to the sticking coefficient to the one that we found previously for the first collision. We show this "rescaled" sticking fraction of the DIP on HMDS in Fig. 3 as a dashed line. [We have no way to determine, using this approach, what the energy of the molecule might be after a third collision or more.]

It is not unreasonable to imagine that experimental sticking coefficients also reflect a similar "ensemble average" of collision energies of DIP with HMDS. Although multiple surface collisions affect the sticking of all the systems we studied, this should be more critical for the stiff HMDS surface than the longer, inherently more flexible, SAM molecules. This expectation is borne out by observing the exit kinetic energy of the DIP molecule (incident energy $7.7 \mathrm{eV}$ ) after colliding with the surface; the exit kinetic energy of the DIP molecule decreases with increasing SAM length (see Fig. 4). Despite the improvement caused by considering the effect of a second, energy-shedding collision with the surface, the disappointing comparison to experimental sticking coefficients for HMDS caused us not to consider this molecule further. Its inclusion here highlights that the sticking coefficient is sensitive to the molecular details of the surface and the strength of molecule-surface binding. Good agreement between experiment and simulation for sticking coefficients of the caliber given in Fig. 3 is not guaranteed.

\section{F. Sticking coefficient of DIP: Effect of packing density of the SAM}

Although experiments typically have little ability to control the density of the SAM (but see [18] as a contrary example), molecular simulations allow us to investigate changes in sticking outcomes as the density is altered. To do so, the room-temperature SAM molecules were packed at different densities ranging from 2.5 molecules $/ \mathrm{nm}^{2}$ to 5.0 molecules $/ \mathrm{nm}^{2}$ (essentially the close-packed limit), studying the DIP-trapping ability of the SAM (i.e., the tendency of DIP to insert or adsorb). This range of densities was chosen to cover an experimentally accessible range.

The FOTS SAM, due to the presence of the larger fluorine atoms, generally requires more "room" on the surface than the OTS SAM. The minimum distance that these molecules can pack together on the surface, $\mathrm{r}_{\text {min }}$, was found to be $6.1 \AA$ for FOTS, while that for its nonfluorinated analog, OTS, was smaller, 5.6 $\AA$. To allow a fairer comparison between the behavior of different SAM molecules, the density of the FOTS SAM matrix was renormalized by the area, defined as the ratio of $r_{\text {min }}$ of the FOTS and the OTS molecules. That is, the density of the FOTS was rescaled to make a fairer comparison to the other two SAMs.

The deposition of a single DIP molecule was simulated, as described earlier, at different densities for a representative incident energy of $7.69 \mathrm{eV}$ onto FOTS, OTS and ODTS SAMs. In terms of total sticking fraction (Fig. 6a), density does not seem to have a pronounced effect in the case of all the SAMs until the density is above about 4.0 molecules $/ \mathrm{nm}^{2}$, at which point the sticking fraction decreases rather sharply with increasing density. Decomposing this result to look at the scattered, inserted and adsorbed fractions as a function of density (Fig. 6), we see two expected results, namely that the tendency to scatter DIP molecules off the SAMs goes up sharply above a density of about 3.5 molecules $/ \mathrm{nm}^{2}$ (as the surface becomes "harder") and that the tendency to insert decreases. The roughly linear decrease in tendency to insert as a function of density might have been more difficult to predict, but the trend is expected. More surprizingly, the fraction of trial depositions that result in molecules adsorbed on the surface at first increases with density up to 3.54.0 molecules $/ \mathrm{nm}^{2}$ and, above that energy, begins to fall. 


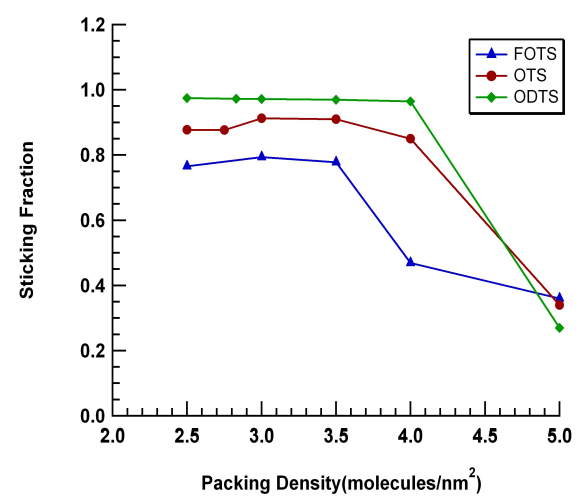

(a) Overall sticking fraction

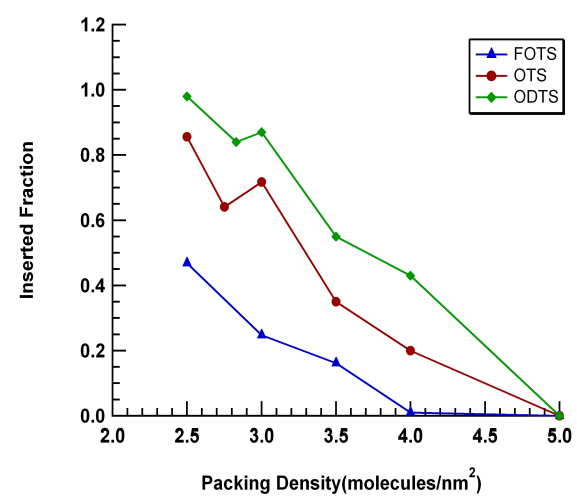

(c) Inserted fraction

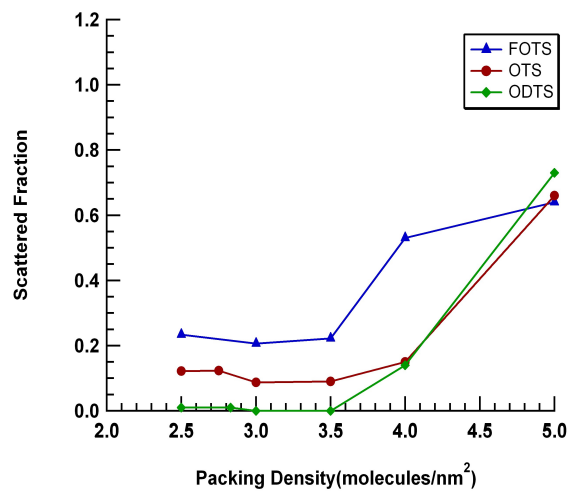

(b) Scattered fraction

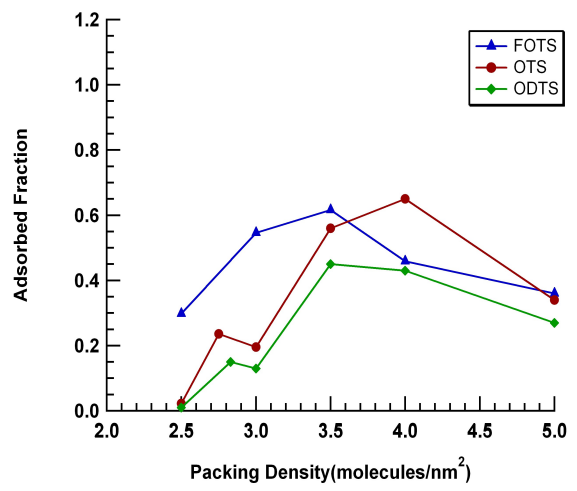

(d) Adsorbed fraction

FIG. 6: Effect of varying the SAM packing density on the tendency to (a) stick, (b) scatter, (c) insert and (d) absorb DIP molecules. Temperature $=300 \mathrm{~K}$. Incident energy $=7.69 \mathrm{eV}$.

Thus, unexpectedly, it appears that there is an optimal SAM packing density to maximize surface adsorption of DIP.

To explain this maximum in sticking fraction with density, consider the following. At low densities, the SAM molecules are quite far apart from one another and do not interact with one another very strongly. Thus, the SAM molecules tend to have greater rotational freedom and wave around their tethering point at the oxygen atom. In this situation, we have seen that it is probable for an incident DIP molecule to collide with only one SAM molecule and insert into the ample space between SAM molecules. The tendency for insertion decreases with increasing density as the SAM molecules become more rotationally constrained by their neighbors' proximity and the incident DIP molecule is most likely to collide with more than one SAM molecule, allowing it to dissipate its kinetic energy more easily and scatter less often. At high densities, the closely packed SAM molecules adopt a more upright stance and lose rotational freedom. The surface loses its capacity to provide a cushioning effect and becomes akin to a "hard" surface. This accounts for higher scattered fractions at higher densities. The two competing effects reach an equilibrium condition at a density of around 3.5 molecules $/ \mathrm{nm}^{2}$ for FOTS in which the adsorbed fraction is highest, and scattering and insertion events are less probable. This maximum is attained at remarkably similar densities, between 3.5 and 4.0 molecules $/ \mathrm{nm}^{2}$, for all three SAMs studied (FOTS, OTS and ODTS). At such densities, the conditions are optimal for DIP molecules to adsorb on the SAM surface.

The density dependence, seen above, also explains an interesting puzzle: The intermolecular interaction energy between a DIP molecule lying on top of the terminal end group of multiple SAM molecules is about $0.1 \mathrm{eV}$ stronger if the SAM is composed of FOTS $(-0.54 \mathrm{eV})$ than with OTS(-0.43 eV); see section II. One might be tempted to imagine that the sticking fraction should thus be greater on FOTS than on OTS, but this is not observed experimentally (or in the simulations shown here). We can now see the reason for this apparent anomaly; the packing density of the SAM is the controlling factor here. The experimentally observed density of FOTS is less than OTS. Higher insertion in OTS leads to an increase in overall sticking fraction on OTS. However, as seen in Fig. 6d, the adsorbed fraction on FOTS is higher than on OTS and ODTS until the maximum adsorption for FOTS is reached. At densities higher than this maximum, the adsorbed fraction on FOTS drops below that of OTS. This is perhaps because of the greater stiffness of the FOTS 


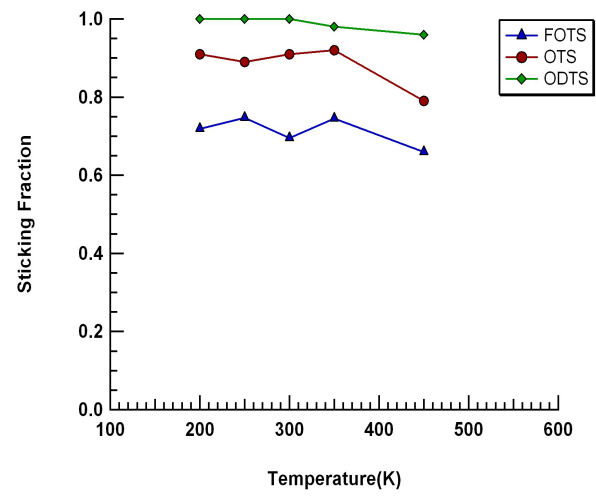

(a) Overall sticking fraction

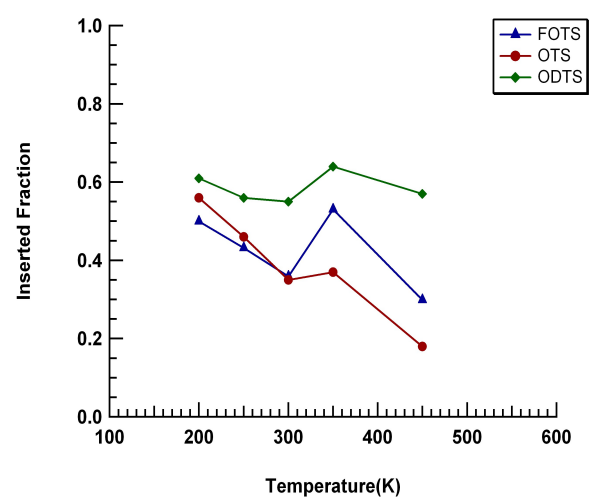

(c) Inserted fraction

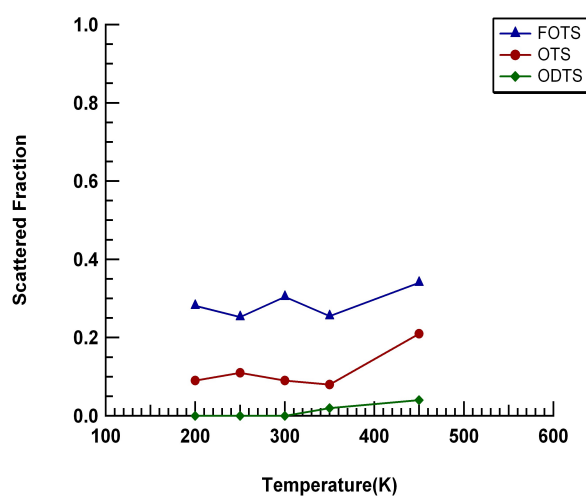

(b) Scattered fraction

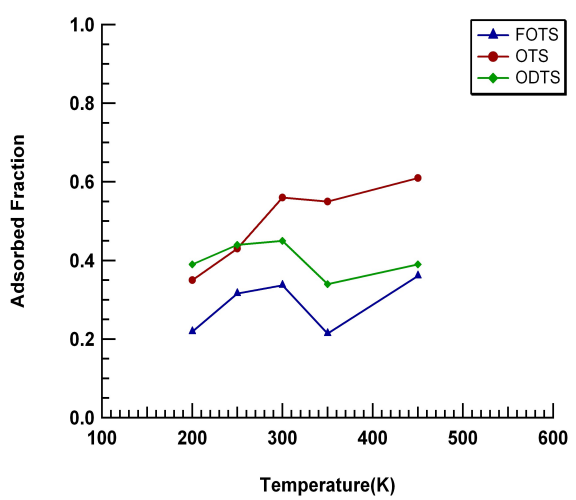

(d) Adsorbed fraction

FIG. 7: Effect of varying the temperature of the SAM surface on the tendency to (a) stick, (b) scatter, (c) insert and (d) adsorb DIP molecules at a density of 3.5 molecules $/ \mathrm{nm}^{2}$ and an energy of $7.69 \mathrm{eV}$.

SAM. The fluorine atoms on FOTS interact strongly with one another, rendering the SAM surface "harder". The same maximum for the OTS and ODTS SAMs occurs at a slightly higher density since these SAMs are "softer." It is interesting to note that maximum adsorption may be achieved on the FOTS SAM at lower packing densities. To achieve the same extent of adsorption on OTS and ODTS would require a higher packing density which is likely to be difficult to obtain experimentally. Thus, by modifying the SAM molecules with suitable substituents, it could become possible to tune the adsorption characteristics of the DIP molecule.

\section{G. Sticking coefficient of DIP: Effect of Temperature}

To investigate the effect of temperature on the ability of DIP to stick/adsorb on SAM surfaces, we thermalized the SAMs at a packing density of 3.5 molecules $/ \mathrm{nm}^{2}$ (near-optimal for surface adsorption) at five different temperatures in an experimentally accessible range ( $\mathrm{T}=$ 200, 250, 300, 350 and $400 \mathrm{~K}$ ). Fig. 7 shows the variation of sticking, scattered, inserted and adsorbed fractions of DIP with temperature. While there was essentially no change observed in the overall sticking fraction of DIP with increasing temperature, we observed that the adsorbed fraction of DIP has a slight tendency to increase as the temperature is increased, for all three SAMs. The inserted fraction consequently decreased and no significant change was observed in the scattered fraction. At low temperatures, the SAM molecules have very little energy and some degrees of freedom are frozen out. This allows the DIP molecule to lose its initial energy into the SAM, but, since the SAM molecules themselves have very low energy at these temperatures, the prevalence of insertion events is very high. The SAM molecules tend to part easily to accomodate the incoming DIP molecule. This accounts for the elevated inserted fraction at low temperatures and lower adsorbed fraction. At higher temperatures, there is an increase in the adsorbed fraction and a decrease in the inserted fraction. At these temperatures, the SAM molecules have greater degrees of freedom and the probability of an insertion event occuring is low because of the extensive vibrational and swaying motion of the SAM molecules as has been previously described. Due to this increased vibrational and disordered motion of the SAM molecules, the probability of surface adsorption of DIP is higher. It may, however, be difficult to conduct experiments at high temperatures since 


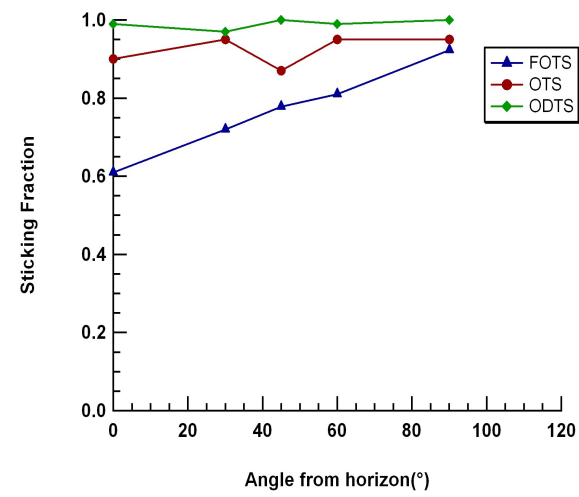

(a) Overall sticking fraction

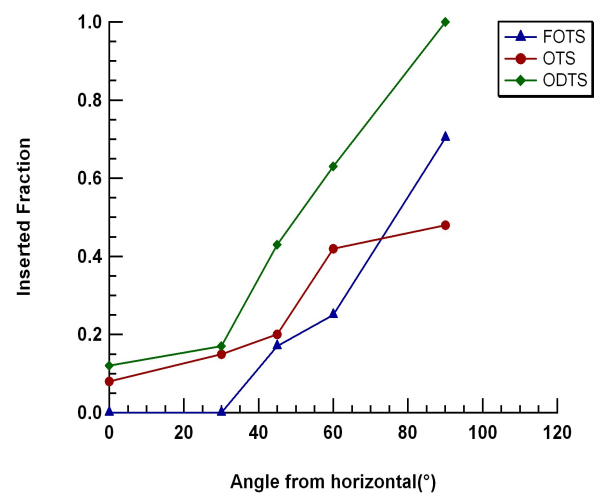

(c) Inserted fraction

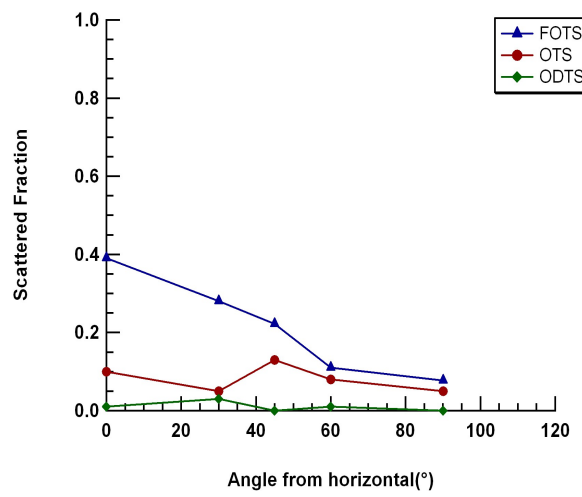

(b) Scattered fraction

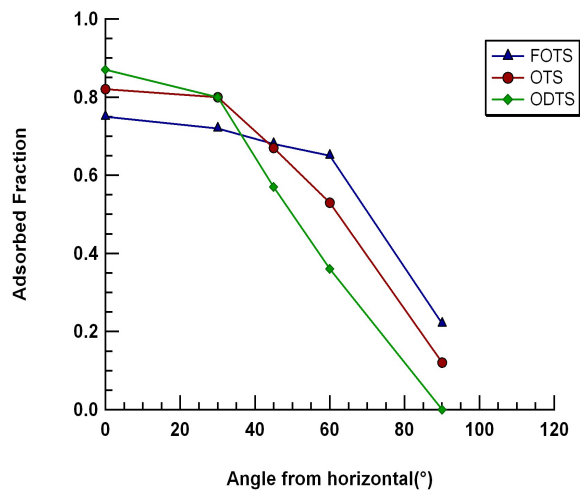

(d) Adsorbed fraction

FIG. 8: Effect of the orientation of the incident DIP molecule on the tendency to (a) stick, (b) scatter, (c) insert or (d) adsorb DIP. Temperature $=300 \mathrm{~K}$. Incident energy $=7.69 \mathrm{eV}$. Packing density $=3.5$ molecules $/ \mathrm{nm}^{2}$.

the SAM molecules desorb from the substrate surface at high temperatures. Thus, room temperature may be optimal for experimental set ups, as is indeed typically the case.

\section{H. Sticking coefficient of DIP: Effect of orientation of the DIP molecule}

All the simulations described above were performed with the DIP molecule initially having a random orientation in space at some point above the surface. To confirm the randomness of the DIP molecules as it strikes the SAM surface, the average angle off-normal of the DIP molecule was calculated to be around $47^{\circ}$, which is roughly midway between a parallel and a perpendicular orientation, confirming the randomness. In this section, we study the effect of specific different orientations of DIP upon the collision dynamics, even though this is currently not possible to emulate in experiments. To do so, we undertook 100 runs with the DIP molecule initially having each of five different orientations with respect to the SAM surface $\left(0,30,45,60\right.$ and $90^{\circ}$ from horizontal). The incident energy of the DIP molecule was set at $7.69 \mathrm{eV}$ for all runs, with the SAMs set at a packing density of 3.5 molecules $/ \mathrm{nm}^{2}$ of the SAMs and a temperature of 300 K. Figs. 8a, 8b, 8c and 8d show the variation of the overall sticking coefficient, and the scattered, inserted and adsorbed fractions of DIP on the SAM surfaces, respectively.

Fig. 8b shows little systematic variation of the scattered fraction as a function of incident angle of the DIP molecule across all three SAMs. In fact, the scattered fraction remains quite small in all three cases, and almost consistently zero in the case of ODTS. This is due to the very flexible nature of ODTS arising because of its length. The longer the molecule, the more flexibility it has at the packing density considered. In contrast, Fig. 8d shows a consistent and dramatic decrease in the adsorbed fraction as a function of incident angle of the DIP molecule; the more grazing the angle of incidence of DIP, the more likely it is to adsorb on the surface. A DIP molecule approaching the surface is able to dissipate incident energy more easily since it can collide with multiple SAM molecules and this orientation offers the strongest van der Waals interactions between the DIP and the SAM molecules (though comparatively still weak in an absolute sense). Thus, the adsorbed fraction in Fig. 8d is seen to steadily decrease from a parallel to a perpendicular orientation. Similarly, we might expect a DIP molecule 
approaching the surface at an angle perpendicular to the surface to be more likely to "slice" through the SAM and increase the probability of insertion events (Fig. 8c), and this is indeed observed. These tendencies counteract one another with the result that the overall sticking fraction becomes only a weak function of angle of incidence, and will differ with the choice of SAM molecule depending on the relative ability to adsorb versus insert.

The fraction of DIP adsorbed on ODTS is greater than that on FOTS for parallel orientations, but is smaller for perpendicular orientations. This is because FOTS molecules appear "harder" to the incoming molecule and tend to scatter a parallel DIP molecule more than a "softer" ODTS molecule. For a perpendicularly inclined incident DIP molecule, the ODTS matrix is more flexible and allows easier insertion than a stiffer FOTS matrix. It may therefore be advisable (if experimentally possible) to have as much of a parallel orientation as possible during deposition to ensure a greater adsorbed fraction of DIP on the SAM, but at low enough incident flux that the surface "shadowing" events that cause roughening and $3 \mathrm{D}$ growth in atomic systems are less of a concern. The role of shadowing in deposition of small-molecule organic semiconductors is largely unexplored.

\section{Interaction Energy of DIP with different SAMs}

It is clear from the results described above that the interaction energy between DIP and the SAM surfaces (relative to the strength of SAM-SAM interactions) plays a role in the adsorption or scattering of the DIP molecule and hence deserved some independent study. To understand this better, we computed the intermolecular interaction energy between a DIP molecule and all the (typically 4-6) SAM molecules that interact with it at the respective densities of the SAMs mentioned in the previous sections. We performed such calculations for three interactions: DIP-SAM in a "T"-configuration redolent of the initial surface adsorption configuration, DIP-SAM in a co-facial configuration characteristic of insertion events, and SAM-SAM interactions to provide us with information about the competition for SAM molecules to prefer the proximity of other SAMs rather than interacting with DIP. The results are summarized in Table II.

For the "T"-configuration we computed the interaction energy as the DIP molecule was moved statically in incremental steps from a position close to the surface to one that was a large distance away from the SAM surface (large enough so that the DIP does not "feel" the SAM surface). The difference between the energy at maximum interaction and the energy at very large distance gives the binding energy of the DIP molecule to the SAM surface. In this idealized T-configuration, the computed binding energies correspond to high SAM density situations. The maximum interaction energy for each DIP-SAM interaction in this " $\mathrm{T}$ "-configuration, representative of surface adsorption, was found to be: FOTS: $-0.5 \mathrm{eV}$, OTS and
ODTS: $-0.4 \mathrm{eV}$ (also see Table II). [OTS and ODTS are identical except for their length and should produce the same binding energy]. At the experimental packing density of the SAM considered here, the DIP in the "T" configuration would be in contact with about 4-5 SAM molecules. Binding energies do not change if we calculate them dynamically at high packing densities of the SAM (like 4.0 molecules $/ \mathrm{nm}^{2}$ ), taking data directly from the MD simulations and averaging them. This largely density-independent interaction energy is because the number of SAM molecules with which the DIP comes in contact in the T-configuration does not change much in the range of packing densities considered. The stronger interaction found for DIP with the FOTS SAM is due to the presence of the fluorine atoms which interact more strongly with the delocalized $\pi$ electron clouds on the DIP. [Note that for the MM3 model used here, this is only taken into account through the phenomenological parameterization fitting process]. Thus, the presence of strong electron-accepting functional groups on the SAM molecules seems to help increase the adsorption probability of DIP.

The presence of fluorine atoms in FOTS also implies a stronger interaction energy between the SAM molecules (SAM-SAM interactions), as proved to be the case: At the density found in Engstrom's experiments, the average interaction energy of a group of 4-6 FOTS molecules (calculated dynamically from 25 ps simulations providing averages over 50,000 configurations) is about $-1.0 \mathrm{eV}$, roughly twice that of a group of OTS molecules, $-0.6 \mathrm{eV}$. The interaction energy (at the experimental density) of a group of ODTS molecules is larger, around $-1.7 \mathrm{eV}$, due to the additional sites on the longer ODTS molecule. These values will increase as the packing density is increased: At a high density of 4.0 molecules $/ \mathrm{nm}^{2}$, for instance, the interaction energy for FOTS is about $-1.9 \mathrm{eV}$, for OTS about $-1.0 \mathrm{eV}$ and for ODTS about $-2.0 \mathrm{eV}$. Due to the waving motion of the SAMs, it is difficult to obtain a constant value for the interaction energy. Thus, all the energies in Table II are quoted to the first decimal place only. Overall, the energy required to separate interacting FOTS molecules is greater than the energy required to separate chains of alkyl groups, such as OTS. This should

TABLE II: Interaction energies (in $\mathrm{eV}$ ) of the DIP in "T" and co-facial configurations with different SAM surfaces. SAM-SAM interaction energies are given for comparison.

\begin{tabular}{cccc}
\hline \hline Configuration & FOTS & OTS & ODTS \\
\hline T- DIP-SAM & -0.5 & -0.4 & -0.4 \\
Co-facial DIP-SAM & -1.8 & -1.5 & -1.7 \\
SAM-SAM low density $^{a}$ & -1.0 & -0.6 & -1.7 \\
SAM-SAM high density $^{b}$ & -1.9 & -1.0 & -2.0 \\
\hline \hline
\end{tabular}

${ }^{a}$ At experimental packing density.

${ }^{b}$ At packing density of 4.0 molecules $/ \mathrm{nm}^{2}$. 
reduce the probability of insertion of DIP molecules between the FOTS SAM molecules and facilitate the growth of smoother films, as is borne out in Fig. 5b.

In a co-facial configuration, the interaction energy (at the experimental density and averaged over 25 ps of MD simulation data) between the DIP and FOTS SAM molecules, i.e., the interaction that occurs once the DIP is inserted into the matrix, is about $-1.8 \mathrm{eV}$. The interaction energy between DIP and OTS is about $-1.5 \mathrm{eV}$, and that between DIP and ODTS is about $-1.7 \mathrm{eV}$. Thus the SAMs we considered give roughly the same value, which (for OTS and ODTS at least) reflects the fact that DIP is interacting with the same chemical environment. The slightly higher value for FOTS is understandable given the more electronegative fluorine atoms. Just as for the T-configuration results described above, these values represent the interaction of one DIP molecule with 5-6 SAM molecules (the average number of SAM molecules with which the DIP comes in contact when inserted into the matrix).

If you compare these results to the SAM-SAM interactions reported above, the co-facial DIP-SAM interactions are greater than the interaction energy between SAM molecules at low density (but become comparable at very high density). From an energetic basis alone, then, it will be preferable for DIP molecules to insert into the SAM matrix (-1.5-1.8 eV for DIP-SAM vs. -0.6 to $-1.0 \mathrm{eV}$ for SAM-SAM), and far more likely than lying on the surface $(-0.4$ to $-0.5 \mathrm{eV})$. This does, indeed, happen in the MD simulations at experimental SAM densities.

Mediating these energetic considerations, entropic and stochastic considerations also play a role in determining the disposition of the DIP molecules to insert versus surface-adsorb. At high packing densities, adsorption is favored. An adsorbed DIP molecule would interact with only the terminal methyl- (or substituted methyl-) group of every SAM molecule. Since the strength of this interaction would be less than when inserted (as mentioned above), the DIP is able to diffuse over the surface of the SAM easily which may lead to formation of a more ordered film if this higher diffusivity contributes to a more $2 \mathrm{D}$ growth. Alternatively, a higher surface diffusion can bring sufficient DIP molecules together for them to spontaneously "flip" upright and form the nucleus for growth of a new ordered layer. Insertion may be prevented for sufficiently high packing densities of the SAM molecules. Thus, we have shown that there are a number of competing processes at play. But barring other factors, higher packing densities seem to be generally more favorable for surface adsorption of DIP and consequently for good film growth.

\section{J. Conclusions}

We have performed thousands of Molecular Dynamics simulations on three different SAM surfaces (and a comparative HMDS surface) to study their propensity to trap DIP molecules incident on the SAM at hyperthermal velocities. The simulations yielded results for a sensitive property, the sticking coefficient, which were in good agreement with experimental data for the three SAMs studied. The results were much less impressive for HMDS, but highlighted the need for surface coatings to have degrees of freedom to dissipate the incident energy during collisions with the depositing material. This agreement illustrated the competency of the chosen intermolecular potential to model DIP and the SAMs studied. The sticking fraction of DIP decreased with increasing energy of incidence suggesting that hyperthermal deposition processes offer no obvious benefit in producing ordered thin films, in line with a complementary experimentally focused companion paper [23]. The molecular scale of the simulations enabled the distinction between adsorbed and inserted fractions of DIP - an important feature that is invariably unobtainable experimentally. A new and unanticipated phenomenon deduced by the simulations was the prediction of an optimal SAM packing density to promote sticking on the surface. While experimental control of the density may be very difficult in practise, this result has implications for the design and choice of SAM molecules to maximize surface adsorption.

We have identified the key factors that govern the sticking fraction of DIP molecules on these SAM surfaces, which we believe can be reasonably expected to carry over to other choices of long alkyl chain SAMs. The more important factors seem to be the chemical functionality of the SAM, the incident energy of the DIP, and the packing density of the SAM - all of which can be controlled experimentally. These results are driven by the balance of binding energies between the SAM molecules and the incoming molecule: T- configuration and co-facial energies compared to SAM-SAM interactions help differentiate surface-binding from insertion tendencies, respectively. Factors such as the temperature of the surface seem less important in governing the deposition characteristics of the DIP molecule. The orientation of the incoming molecule is capable of strongly affecting the tendency to adsorb on the surface, but is essentially not experimentally controllable. Overall, grazing angles favor surface-adsorption and normal deposition facilitates insertion, but this is also dependent on the nature of the SAM. There is little or no experimental or simulation studies of the effect of grazing incidence on the nature of the grown film for small-molecule organic semiconductors. It would be interesting to compare the role of shadowing for simple monatomic systems that causes oriented dendritic growth to that for small-molecule organics in which the highly anisotropic interactions may disrupt or enhance this tendency.

While we were able to explain a lot of the observed tendency to insert versus surface-adsorb in terms of the energetics of binding energies between DIP and SAM in comparison to SAM-SAM and DIP-DIP interactions, we noted that energetics alone do not control this complex process. Stochastic and entropically driven processes also 
play a role.

Since we are able to predict the behavior of the deposition of DIP based on the factors above, computation can be used to design a suitable SAM surface which possesses the properties necessary to attain high adsorption of DIP on the surface. However, intelligently designing an optimized SAM surface computationally can present significant challenges of its own. For instance, the behavior of different organic semiconducting molecules such as the conformationally rich rubrene molecules, or ones that differ considerably in shape, like $\mathrm{C}_{60}$, needs to be examined to determine whether their deposition behavior is similar to that for the DIP molecules studied here. The behavior of similarly shaped, relatively rigid, acenes and perhaps the phenyls (biphenyl to sexiphenyl, say) might be expected to behave similarly to DIP.

The present study has not examined the effect on sticking coefficient of the presence of other DIP molecules on the surface (from preceding deposition events) which is bound to affect the quality of subsequent film growth. Examining the effects of depositing a large number of DIP molecules on a SAM surface is computationally expensive but it is the next logical step towards computational studies following the growth of thin films of smallmolecule organic semiconductors.

\section{Acknowledgments}

This publication was based on work supported by Award No. KUS-C1-018-02, made by King Abdullah University of Science and Technology (KAUST). The Engstrom research group at Cornell are thanked for access to their experimental data in advance of publication. Intel Corporation is thanked for the donation of computing resources.
[1] H. Yamamoto, K. Nishiyama, and I. Ohdomari, J. Phys. IV 132, 189 (2006).

[2] L. Zuppiroli, K. Kamaras, M. Bussac, and E. Moons, Eur. Phys. J. B 11, 505 (1999).

[3] M. Halik, H. Klauk, , and U. e. a. Zschieschang, Nature 431, 963,966 (2004).

[4] D. L. Irving and D. W. Brenner, J. Phys. Chem. B 110, 31 (2006).

[5] S. Vemparala, B. B. Karki, R. K. Kalia, and P. Vashishta, J. Chem. Phys. 121(9), 4323 (2004).

[6] N. Allinger, Y. Yuh, and J.-H. Lii, J. Am. Chem. Soc. 111, 8551 (1989).

[7] K.-H. Chen, J.-H. Lii, G. A. Walker, Y. Xie, H. F. S. III, and N. L. Allinger, J. Phys. Chem. A. 110, 7202,7227 (2006).

[8] K.-H. Chen, G. A. Walker, and N. L. Allinger, Journal of Molecular Structure(Theochem) 490, 87,107 (1999).

[9] J. E. Goose and P. Clancy, J. Phys. Chem. C 111, 43 (2007).

[10] R. Cantrell and P. Clancy, Surface Science 602(22), 3499 (2008).

[11] S. B. M. Bosio and W. L. Hase, J. Chem. Phys. 107, 9677 (1997).

[12] N. Isa, K. D. Gibson, T. Yan, W. L. Hase, and S. J. Sibener, J. Chem. Phys. 120, 2417 (2004).

[13] B. S. Day, J. M. Morris, W. A. Alexander, and D. Troya, J. Phys. Chem. B 110, 6832 (2006).

[14] B. S. Day, J. M. Morris, and D. Troya, J. Chem. Phys. 122, $214712(2005)$.
[15] K. D. Gibson, N. Isa, and N. Sibener, J. Phys. Chem. A 110, 1469 (2006).

[16] A. Ulman, Adv. Mater. 212 (1990).

[17] D. H. L. Kim, H. S. Yang, H. Yang, and K. L. Cho, Advanced Functional Materials 18, 1363,1370 (2008).

[18] Y. Ito, A. A. Virkar, S. Mannsfeld, J. H. Oh, M. Toney, J. Locklin, and Z. Bao, J. Am. Chem. Soc. 131, 9396,9404 (2009).

[19] F. Tao and S. L. Bernasek, Chem. Rev. 107, 1408,1453 (2007).

[20] P. T. Mikulski, L. A. Herman, and J. A. Harrison, Langmuir 21, 12197,12206 (2005).

[21] S. Hong, A. Amassian, A. Woll, S. Bhargava, J. Ferguson, G. Malliaras, J. Brock, and J. Engstrom, Appl. Phys. Lett. 92, 253304 (2008).

[22] A. Amassian, T. Desai, S. Kowarik, S. Hong, A. R. Woll, G. Malliaras, F. Schreiber, and J. Engstrom, J. Chem. Phys. 130, 124701 (2009).

[23] T. V. Desai, S. Hong, A. R. Woll, K. J. Hughes, A. Prakash, P. Clancy, and J. R. Engstrom, submitted (2010).

[24] G.Schaftenaar and J. Noordik, J. Comput.Aided Mol. Design 14, 123,134 (2000).

[25] J. W. Ponder, Tinker - software tools for molecular design, http://dasher.wustl.edu/tinker/ (2010).

[26] J. E. Goose, E. L. First, and P. Clancy, Phys. Rev. B 81, 205310 (2010).

[27] J. Nocedal, Mathematics of Computation 35, 773 (1980). 